\title{
Perfil epidemiológico de idosos hipertensos no Brasil: uma revisão integrativa
}

\author{
Epidemiogical profile of hypertensives in the elderly in Brazil: an integrative review
}

\begin{abstract}
Álef Lamark Alves Bezerra ${ }^{1}$, Daniel Sarmento Bezerra ${ }^{2}$, Danielle Serafim Pinto ${ }^{3}$, André Ricardo Bezerra Bonzi ${ }^{4}$, Ricardo Montenegro Nóbrega de Pontes ${ }^{5}$ José Artur de Paiva Veloso $^{6}$
\end{abstract}

Bezerra ALA, Bezerra SA, Pinto DS, Bonzi ARB, Pontes RMN, Veloso JAP. Perfil epidemiológico de idosos hipertensos no Brasil: uma revisão integrativa / Epidemiological profile of hypertensives in the elderly in Brazil: an integrative review. Rev Med (São Paulo). 2018 jan.-fev.;97(1):103-7.

RESUMO: O aumento da população idosa tem consequências diretas sobre os sistemas de saúde pública, uma vez que a velhice está mais intimamente ligada a condições patológicas que comprometem alguns sistemas, como o sistema vascular, por exemplo. Assim, com o envelhecimento, o indivíduo é suscetível a mais morbidades como a hipertensão, que é destacada como fator de risco para a qualidade de vida e declínio na função cognitiva. Por isso, realizou-se este estudo cujo objetivo é descrever o perfil epidemiológico de pacientes hipertensos e idosos matriculados no programa Hiperdia e residentes em estados do Brasil. Foi analisada sua associação com os principais fatores de risco como o tabagismo, o sedentarismo e o excesso de peso. Trata-se de uma revisão integrativa da literatura, composta por publicações relativas aos anos de 2012 a maio de 2016, em português, inglês ou espanhol, indexadas do portal da Biblioteca Virtual de Saúde, BVS, nos seguintes bancos de dados: LILACS; BDENF, MEDLINE e SciELO. A hipertensão é comum nos idosos e tem a sua prevalência aumentada pelo envelhecimento, condicionando assim a morbidade e a mortalidade desta população. Entre os principais fatores de risco encontrados em pesquisa e estudos estão a alta circunferência da cintura, o excesso de peso e, muitas vezes, o consumo de gorduras saturadas. Além destes, estão o hábito de fumar, beber álcool e fazer exercício de forma irregular. Cabe citar que a maioria dos estudos sugere que as mulheres mais velhas são o público mais danificado. Finalmente, o programa Hiperdia é decisivo para melhorar a qualidade de vida, pois faz a regulamentação do atendimento médico e fornece tanto controle quanto sinais para problemas que podem ocorrer dentro da população assistida e deve haver de parte da sociedade e do estado e esforço para aprimorar este programa.

Descritores: Assistência integral a saúde; Saúde do idoso; Hipertensão/epidemiologia; Fatores de risco; Perfil de saúde; Brasil/epidemiologia.

ABSTRACT: The increase in the elderly population has direct consequences on public health systems, since old age is more closely linked to pathological conditions that compromise some systems, such as the vascular system, for example. Thus, with aging, the individual is susceptible to more morbidities such as hypertension, which is highlighted as a risk factor for quality of life and decline in cognitive function. Therefore, the purpose of this study was to describe the epidemiological profile of hypertensive and elderly patients enrolled in the Hiperdia program and residents of Brazilian states. Its association with the main risk factors such as smoking, sedentary lifestyle and overweight were analyzed. This is an integrative review of the literature, composed of publications related to the years 2012 to

1. Faculdade de Ciências Médicas da Paraíba. Discente. E-mail: aleflamark@gmail.com, https://orcid.org/0000-0001-9294-1796.

2. Faculdade de Medicina Nova Esperança. Aluno do Curso de Medicina. E-mail: sarmentomeddaniel@gmail.com, https://orcid.org/0000-0003-1430-4796.

3. Faculdade de Medicina Nova Esperança. Farmacêutica pela Universidade Federal da Paraíba - UFPB, doutora em Produtos Naturais e Sintéticos Bioativos pela UFPB; docente e. Coordenadora do Curso de Medicina. E-mail: dani-serafim@hotmail.com, https://orcid.org/0000-0002-9961-7558

4. Faculdade Maurício de Nassau João Pessoa. Discente. E-mail: bonzipb@gmail.com, https://orcid.org/0000-0001-5323-4095.

5. Faculdade de Ciências Médicas da Paraíba. Médico. e-mail: ricardomontenegro2@yahoo.com.br, https://orcid.org/0000-0001-8328-5387

6. Faculdade de Ciências Médicas da Paraíba. Graduado em Fisioterapia. Docente. e-mail: arturvelosofisio@gmail.com, https://orcid.org/0000-00018606-5953.

Endereço para correspondência: Daniel Sarmento Bezerra. End.: Avenida Sergipe, 311, Bairro dos Estados. João Pessoa, PB. CEP: 58030-190. E-mail: sarmentomeddaniel@gmail.com. 
May 2016, in Portuguese, English or Spanish, indexed from the Virtual Health Library portal, VHL, in the following databases: LILACS; BDENF, MEDLINE and SCIELO. Hypertension is common in the elderly and its prevalence is increased by aging, thus conditioning the morbidity and mortality of this population. Among the major risk factors found in research and studies are the high waist circumference, overweight and often the consumption of saturated fats. In addition to these, there is the habit of smoking, drinking alcohol and doing irregular exercise. It should be noted that most studies suggest that older women are the most impaired

\section{INTRODUÇÃO}

Qumento da população idosa é um fenômeno irreversível e natural e que possui consequências diretas nos sistemas de saúde pública do Brasil e do mundo. O envelhecimento populacional está relacionado a processos paralelos, como a diminuição das taxas de fecundidade e natalidade, aumento progressivo da expectativa de vida, necessidade de adaptação ao avanço tecnológico e o acesso aos serviços de saúde, dentre outros. Além disso, é um processo de transformação da estrutura física e cognitiva; bem como da percepção subjetiva de tudo que cerca o ser humano ${ }^{1}$.

A velhice gera alterações no cotidiano e que, em casos pontuais, acometem a autonomia da pessoa idosa, principalmente quando o processo vem acompanhado de condições patológicas que comprometem sistemas como o vascular e o nervoso. Dessa forma, com o avançar da idade morbidades típicas e irremediavelmente prevalentes da faixa etária anunciam-se, dentre estas diabetes mellitus (DM), doenças isquêmicas do coração, doenças do sistema circulatório, doenças cerebrovasculares e hipertensão $\operatorname{arterial}^{2,3}$.

A Hipertensão Arterial Sistêmica (HAS) é destacada como fator de risco para o declínio da função cognitiva como também para o declínio da qualidade de vida do idoso, estando entre os maiores problemas de saúde pública. Ela se apresenta de maneira multifatorial e se caracteriza por apresentar níveis elevados e rotineiros de pressão arterial sistólica maiores que $140 \mathrm{mmHg}$ ou pressão arterial diastólica maiores que $90 \mathrm{mmHg}^{4,5}$. Vale frisar que ela também é importante fator de risco de morbimortalidade cardiovascular, e, no Brasil, atinge $25 \%$ da população com mais de 20 anos.

Tanto os infartos do miocárdio locais ou bilaterais como as lesões em substância branca provocam a desmielinização e consequente estreitamento da luz do vaso, o que está associado tanto HAS quanto a disfunção cognitiva. Ademais, o controle da hipertensão arterial através de medicamentos anti-hipertensivos configura como importante adjuvante na prevenção ao declínio cognitivo, uma vez que protege dos efeitos que recaem sobre a substância branca cerebral e também vascular; sendo a adesão ao tratamento decisivo para o sucesso do tratamento ${ }^{1,5}$. public. Finally, the Hiperdia program is decisive for improving the quality of life, since it regulates medical care and provides both control and signals for problems that may occur within the assisted population and must be from the society and the state and efforts to improve this program.

Keywords: Comprehensive health care; Health of the elderly; Hypertension/epidemiology; Risk factors; Health profile; Brazil/ epidemiology.

Dentre os principais fatores de risco encontrados em pesquisas e estudos estão à circunferência elevada da cintura, o excesso de peso e o consumo de gorduras saturadas com frequência; além disso, os pacientes costumam ter maus hábitos como fumar, consumir bebidas alcoólicas e não fazer exercícios físicos regularmente ${ }^{6}$.

O Programa HIPERDIA - Programa Nacional de Hipertensão e Diabetes Mellitus do Ministério da Saúde - inscreve, acompanha e avalia pacientes hipertensos e/ ou diabéticos, com a finalidade de fazer uma intervenção ativa sobre esta população e traçar metas, possuindo, para isto, um banco de dados detalhado por faixa etária, sexo, medicamentos utilizados e medição da pressão arterial. A alimentação dos dados do banco supracitado é de responsabilidade dos departamentos de saúde das cidades de cada estado brasileiro ${ }^{5}$.

Nesse contexto, o objetivo deste estudo é descrever o perfil epidemiológico de pacientes hipertensos e idosos, inscritos no Programa Hiperdia, residentes no Brasil, analisando sua associação com os principais fatores de risco, como tabagismo, sedentarismo e sobrepeso.

\section{METODOLOGIA}

Trata-se de uma revisão integrativa da literatura, constituída por publicações indexadas a partir do portal da BVS (Biblioteca Virtual de Saúde), nas seguintes bases de dados: LILACS (Literatura Latino-americana e do Caribe em Ciências Sociais e da Saúde); BDENF (Banco de Dados de Enfermagem), MEDLINE (Medical Literature Analysis and Retrieval System on-line) e também na SciELO (Scientific Eletronic Library Online). Essa busca foi realizada no período de julho de 2016, utilizando a terminologia em saúde, consultada nos Descritores em Ciências da Saúde (DeCS/Bireme), identificando os termos no idioma português: HIPERDIA e fatores de risco e idosos.

Para o refinamento da pesquisa, foram definidos como critérios de inclusão: artigos que abordassem sobre hipertensão arterial e idoso, indexados nas bases de dados escolhidas para o estudo e publicados entre o período de 2012 a maio de 2016, nos idiomas inglês, português e espanhol. Os critérios de exclusão foram: artigos incompletos, relacionados à pesquisa metodológica, capítulos de livros, dissertações, teses e trabalhos que se tratava de descrição de eventos. O texto completo dos 
artigos foi obtido on-line. Iniciada a pesquisa, foram encontrados 35.674 artigos e após filtro, do período entre 2012 a maio de 2016, e selecionar Brasil como país do assunto, restaram 181 artigos. Os artigos restantes foram organizados e arquivados em pastas denominadas de acordo com a base de dados em que foram localizados. Em seguida foi feito a seleção daqueles que faziam referência ao objeto de estudo restando 50 trabalhos dos quais, 15 foram excluídos por estarem repetidos nas bases de dados LILACS, MEDLINE, BDENF. Após a leitura dos resumos dos 35 artigos restantes, 15 foram excluídos, por não atenderem aos critérios de inclusão estabelecidos para o estudo e 5 por não apresentar respostas para a questão norteadora da pesquisa e os objetivos propostos para este estudo. Em seguida, procedeu-se à leitura na íntegra dos 10 artigos que constituíram o corpus da revisão integrativa.

Neste sentido, o artigo compõe uma revisão crítica que não se ocupa somente em trazer um apanhado de ideias sobre a temática, mas também estabelecer o diálogo entre os autores referenciados, na perspectiva de fazer uma discussão sobre o perfil epidemiológico de pacientes hipertensos e idosos, inscritos no Programa Hiperdia, residentes em estados do Brasil, descrevendo a importância dos hábitos saudáveis e o consequente aumento da qualidade de vida dos mesmos.

\section{DISCUSSÃO E RESULTADOS}

Das 10 publicações encontradas, observou-se que o maior número de publicações ocorreu no ano de 2015, tendo um total de 5 publicações. Seguido dele vem empatados os anos de 2013 e 2014, cada um com duas publicações e por fim o ano de 2012 com apenas uma publicação; os anos anteriores a 2012 não foram selecionados. Em relação aos periódicos, destacaram-se importantes revistas, dentre as quais merecem evidência a "Revista Gaúcha de Enfermagem" e a revista "Epidemiologia e Serviços de Saúde" conforme indicado na Tabela 1.

Tabela 1 - Distribuição dos artigos sobre HIPERDIA e idosos e fatores de risco, segundo os periódicos científicos. João PessoaPB-Brasil, 2016

\begin{tabular}{l|l}
\hline Revista & $\mathbf{N}^{\mathbf{0}}$ \\
\hline Revista Brasileira de Geriatria e Gerontologia. & 3 \\
\hline Caderno de Saúde Coletiva. & 3 \\
\hline Revista Kairós Gerontologia & 1 \\
\hline Arquivo Brasileiro de Cardiologia. & 2 \\
\hline Revista Mineira de Enfermagem & 1 \\
\hline
\end{tabular}

Fonte: Própria pesquisa.

No Quadro 1 são ilustrados o título dos artigos, seus objetivos e resultados.

Um estudo $^{4}$ feito no município de Marques de Souza, no Rio Grande do Sul, relata que a hipertensão arterial constitui grave problema de saúde pública, evidenciando os idosos como a grande maioria dos pacientes. $\mathrm{O}$ estudo supracitado relata que a pressão arterial aumenta com a idade de forma gradual e contínua. Ou seja, a HAS é comum em idosos e tem sua prevalência aumentada pelo envelhecimento, dessa maneira condicionando a morbimortalidade dessa população. Além disso, com o passar da idade, as pessoas ficam mais sujeitas a outros fatores de risco como o excesso de peso, a circunferência da cintura elevada e o enrijecimento arterial.

Segundo pesquisas ${ }^{4,7}$ na cidade de Blumenau/SC, $70,4 \%$ das pessoas inscritas no programa HIPERDIA são portadoras de HAS. Um fator importante para a redução e controle desta condição é o tratamento adequado e a mudança dos hábitos alimentares. A não adesão ao tratamento medicamentoso implica em complicações clínicas e maiores custos a saúde pública do país. Ainda, é preocupante a questão de que vários estudos, em diferentes regiões do país, demonstram que a população possui pouca informação sobre a medicação que faz uso, além de também se evidenciar o mau gerenciamento do tratamento pelo paciente ${ }^{8}$. Ademais, o aumento de peso, o tabagismo e o alcoolismo contribuem para o aumento da pressão arterial, tendo os pacientes de menor escolaridade maiores chances de desenvolver o excesso de peso e, por consequência, o descontrole da pressão ${ }^{4}$. No entanto, no que se remete ao peso, existe um estudo que discorda e traz o aumento de peso como fator de risco irrelevante ${ }^{9}$. O que reforça a necessidade de maiores e diversificados estudos para se chegar a uma maior conclusão sobre o tema.

Embora as doenças crônicas não transmissíveis sejam mais predominantes nas faixas etárias mais avançadas, observa-se, ao mesmo tempo que a desnutrição diminuiu, e, ademais, observa-se que as taxas de obesidade se elevou nessa população. Esta característica marca a transição nutricional no país outrora exclusivo de áreas urbanas. Por outro lado, a inclusão de atividade física regular aliada a uma transformação de hábitos alimentares, onde ocorra a diminuição do consumo de produtos industrializados, ricos em açúcares e gorduras; e consequentemente a introdução de uma alimentação variada, de alto valor nutricional, mostrasse condição essencial e definitiva para a redução no número de hipertensos como também para o aumento da qualidade de vida dessa população ${ }^{6}$.

Estudos paralelos envolvendo a capacidade funcional dos idosos, como o desenvolvido em Belém do Pará, são importantes indicadores de saúde e qualidade de vida, além de auxiliar o trabalho dos profissionais que atendem pacientes do HIPERDIA, uma vez que traz informações adicionais acerca de riscos e agravos a saúde daqueles já acompanhados pelo programa ${ }^{10}$. Na Paraíba, dados coletados através do Sistema de Cadastramento e Acompanhamento de Hipertensos e Diabéticos, demonstram a porcentagem implicada nos principais fatores de risco, entre o período de janeiro de $2009-$ abril de 2013, envolvendo idosos de ambos os sexos. Os resultados estão descritos no quadro abaixo, onde destacase o sedentarismo feminino, mas cabe citar que além do sedentarismo, o tabagismo e o sobrepeso também são fatores de risco. 
Bezerra ALA, et al. Perfil epidemiológico de idosos hipertensos no Brasil.

Quadro 1 - Detalhamento dos artigos analisados de acordo com o título, os objetivos do estudo e resultados

\begin{tabular}{|c|c|c|c|}
\hline ID & Titulo & Objetivos & Resultados \\
\hline $\mathrm{A} 1$ & $\begin{array}{l}\text { Associação entre declínio cognitivo } \\
\text { e qualidade de vida de idosos } \\
\text { hipertensos. }\end{array}$ & $\begin{array}{l}\text { Analisar a associação entre o declínio cognitivo } \\
\text { e a qualidade de vida de idosos hipertensos. }\end{array}$ & $\begin{array}{l}\text { A prevalência de declínio cognitivo foi de } 20,80 \% \text {, com predominância } \\
\text { em idosos com baixa escolaridade ( } 45,83 \%) \text {. Idosos hipertensos com } \\
\text { declínio cognitivo apresentaram pior qualidade de vida, comparados } \\
\text { aos idosos hipertensos sem declínio cognitivo. Houve associação } \\
\text { positiva da função cognitiva com a qualidade de vida }\end{array}$ \\
\hline A2 & $\begin{array}{l}\text { Fatores associados à hipertensão } \\
\text { arterial sistêmica e ao estado } \\
\text { nutricional de hipertensos inscritos } \\
\text { no programa Hiperdia. }\end{array}$ & $\begin{array}{l}\text { Analisar fatores associados à Hipertensão } \\
\text { Arterial Sistêmica (HAS) e sua relação com } \\
\text { o estado nutricional de indivíduos hipertensos } \\
\text { cadastrados no programa Hiperdia em um } \\
\text { município do Rio Grande do Sul. }\end{array}$ & $\begin{array}{l}\text { Entre os } 402 \text { hipertensos estudados, } 138(34,3 \%) \text { eram adultos e } 264 \\
(65,7 \%) \text { eram idosos, sendo que a maioria era do gênero feminino. } \\
\text { A prevalência de excesso de peso foi de } 76,8 \% \text { em adultos enquanto } \\
35 \% \text { dos idosos apresentaram peso normal. Quando comparadas as } \\
\text { faixas etárias, } 11 \% \text { dos idosos estavam significativamente associados } \\
\text { ao analfabetismo. Os adultos e idosos apresentaram média de } \\
\text { circunferência da cintura e classificação do IMC acima dos níveis } \\
\text { desejados. }\end{array}$ \\
\hline $\mathrm{A} 3$ & $\begin{array}{l}\text { Capacidade funcional de idosos } \\
\text { atendidos em um programa do SUS } \\
\text { em Belém (PA). }\end{array}$ & $\begin{array}{l}\text { O objetivo deste estudo foi avaliar fatores } \\
\text { associados à capacidade funcional em idosos } \\
\text { atendidos em um programa de saúde pública - } \\
\text { Hiperdia - em Belém (PA). }\end{array}$ & $\begin{array}{l}\text { Os idosos tinham em média } 68,3 \text { anos }(\mathrm{DP} \pm 6,2) \text { e } 71,0 \% \text { eram } \\
\text { do sexo feminino. Após ajustes por variáveis de confundimento, } \\
\text { observaram-se maiores chances de pior desempenho no teste entre as } \\
\text { mulheres, os mais velhos e os de menor escolaridade, além de queixa } \\
\text { de dor no joelho, diagnóstico anterior de catarata e autoavaliação de } \\
\text { saúde regular/ruim. }\end{array}$ \\
\hline A4 & $\begin{array}{l}\text { A desão a o tratamento } \\
\text { medicamentoso em usuários } \\
\text { cadastrados no Programa Hiperdia } \\
\text { no município de Teresina (PI). }\end{array}$ & $\begin{array}{l}\text { Avaliar o perfil socioeconômico e a adesão } \\
\text { terapêutica dos usuários cadastrados no } \\
\text { Programa Hiperdia no município de Teresina } \\
\text { (PI). }\end{array}$ & $\begin{array}{l}\text { Não houve associação entre as variáveis sociodemográficas-clínicas } \\
\text { e a adesão segundo Morisky-Green, no entanto houve associação } \\
\text { com o tipo de morbidade apresentada. Diante desses resultados, a } \\
\text { educação em saúde torna-se a principal estratégia para melhorar a } \\
\text { adesão dos usuários, como também a participação de profissionais } \\
\text { na Equipe Saúde da Família, como: o farmacêutico, o nutricionista } \\
\text { e o educador físico }\end{array}$ \\
\hline A5 & $\begin{array}{l}\text { Fatores de risco e proteção para } \\
\text { o desenvolvimento de doenças } \\
\text { crônicas em população idosa rural } \\
\text { do Rio Grande do Sul. }\end{array}$ & $\begin{array}{l}\text { Investigar a presença de fatores de risco e } \\
\text { proteção para doenças crônicas na população } \\
\text { idosa rural da Linha Senador Ramiro do } \\
\text { município de Nova Bassano, RS. }\end{array}$ & $\begin{array}{l}\text { Os fatores de risco encontrados foram o elevado consumo de gordura } \\
\text { saturada, excesso de peso e elevada CC. Já os de proteção foram } \\
\text { consumo regular de frutas e verduras por grande parte da população, } \\
\text { baixo sedentarismo, consumo moderado e regular de vinho, baixo } \\
\text { tabagismo e a realização de exames preventivos pelo sexo feminino. }\end{array}$ \\
\hline A6 & $\begin{array}{l}\text { Blood Pressure Control in } \\
\text { Hypertensive Patients in the } \\
\text { "Hiperdia Program": A Territory- } \\
\text { Based Study. }\end{array}$ & $\begin{array}{l}\text { Descrever o perfil epidemiológico e avaliar } \\
\text { o controle da pressão arterial de pacientes } \\
\text { inscritos no Hiperdia, na cidade de Novo } \\
\text { Hamburgo (Estado do Rio Grande do Sul). }\end{array}$ & $\begin{array}{l}\text { Mesmo para pacientes hipertensos inscritos no Programa Hiperdia, } \\
\text { controle da pressão arterial não é satisfatoriamente atingido ou } \\
\text { mantido. pacientes hipertensos diabéticos apresentam o mais } \\
\text { inadequado BP controle. }\end{array}$ \\
\hline A7 & $\begin{array}{l}\text { As contribuições do cuidado ao } \\
\text { idoso no programa de HIPERDIA, } \\
\text { para a formação profissional. }\end{array}$ & $\begin{array}{l}\text { Este estudo objetiva relatar de forma descritiva } \\
\text { a experiência vivenciada na unidade básica } \\
\text { de saúde por acadêmicos, e descrever as } \\
\text { práticas desenvolvidas ao idoso no programa } \\
\text { HIPERDIA, contribuindo para a formação } \\
\text { profissional desses acadêmicos. }\end{array}$ & $\begin{array}{l}\text { O estudo permitiu constatar que, para a formação do acadêmico } \\
\text { de enfermagem, o estágio curricular e extracurricular é de grande } \\
\text { importância, pois possibilita a junção da teoria e prática, por meio das } \\
\text { atividades que são realizadas durante esses estágios. }\end{array}$ \\
\hline A8 & $\begin{array}{l}\text { Percepção de idosos acerca das } \\
\text { atividades desenvolvidas no } \\
\text { hiperdia. }\end{array}$ & $\begin{array}{l}\text { Conhecer a percepção de pessoas com } \\
\text { hipertensão acerca da assistência recebida na } \\
\text { atenção primária. }\end{array}$ & $\begin{array}{l}\text { Atividades do HIPERDIA focadas na entrega de medicamentos: duas } \\
\text { faces de uma mesma moeda; as lacunas e dificuldades no HIPERDIA; } \\
\text { A boa relação com profissionais de saúde gera satisfação com o serviço. }\end{array}$ \\
\hline A9 & $\begin{array}{l}\text { Variações na Prevalência dos } \\
\text { Fatores de Risco para Doença } \\
\text { Arterial Coronariana no Rio } \\
\text { Grande do Sul: Uma Análise } \\
\text { Comparativa entre 2002-2014. }\end{array}$ & $\begin{array}{l}\text { Conhecer a prevalência dos FR da DAC em } \\
\text { cidades do Estado do Rio Grande do Sul, e } \\
\text { compará-la com a encontrada em pesquisa } \\
\text { semelhante realizada nas mesmas cidades } \\
\text { em } 2002 \text {. }\end{array}$ & $\begin{array}{l}\text { A amostra foi constituída de } 26 \% \text { de idosos e } 57 \% \text { de mulheres. } \\
\text { As prevalências dos FR foram de } 44 \% \text { de sedentarismo, } 50 \% \text { de } \\
\text { antecedentes familiares, } 23 \% \text { de tabagismo, } 68 \% \text { de sobrepeso/ } \\
\text { obesidade, } 43 \% \text { de dislipidemia (colesterol elevado), } 40 \% \text { de HAS, e } \\
11 \% \text { de diabetes. Quando comparado ao estudo de } 2002 \text {, verificou-se } \\
\text { que a população de fumantes ativos e sedentários diminuiu, e a de } \\
\text { hipertensos, dislipidêmicos e obesos aumentou. A obesidade é o FR } \\
\text { mais prevalente em mulheres, e a HAS o mais prevalente em homens. }\end{array}$ \\
\hline A10 & $\begin{array}{l}\text { Prevalência de fatores de risco } \\
\text { cardiovascular em idosos não } \\
\text { institucionalizados. }\end{array}$ & $\begin{array}{l}\text { Descrever as prevalências de fatores de risco } \\
\text { para doenças cardiovasculares em idosos não } \\
\text { institucionalizados, conforme sexo e grupo } \\
\text { etário. }\end{array}$ & $\begin{array}{l}\text { As prevalências de hipertensão arterial, hipercolesterolemia, excesso } \\
\text { de peso e adiposidade abdominal foram, respectivamente, de } 82,30 \% \\
\text { para idosas e } 76,81 \% \text { para idosos }(p>0,05), 43,64 \% \text { para idosas e } \\
25,48 \% \text { para idosos }(p<0,05), 47,40 \% \text { para idosas e } 36,12 \% \text { para } \\
\text { idosos }(p<0,05 \text { ) e de } 76,16 \% \text { para idosas e } 41,06 \% \text { para idosos } \\
\text { (p<0,05). A respeito das diferenças por grupo etário, identificou-se } \\
\text { menor prevalência, somente para o excesso de peso, à medida que } \\
\text { a idade avança. }\end{array}$ \\
\hline
\end{tabular}

Fonte: Própria pesquisa. 
Bezerra ALA, et al. Perfil epidemiológico de idosos hipertensos no Brasil.

Quadro 3 - Detalhamento dos idosos acometidos por hipertensão e diabetes com os principais fatores de risco associados

\begin{tabular}{|l|c|c|c|}
\hline & Tabagismo & Sedentarismo & Sobrepeso \\
\hline Masc. & $6,04 \%$ & $17,06 \%$ & $9,6 \%$ \\
\hline Fem. & $10,8 \%$ & $34,66 \%$ & $21,79 \%$ \\
\hline
\end{tabular}

Fonte: Sistema de Cadastramento e Acompanhamento de Hipertensos e Diabéticos - PB.

\section{CONSIDERAÇÕES FINAIS}

As atividades do programa HIPERDIA visam estimular a mudança de hábitos da população assistida; inclusive, pensando e repensando estratégias para aumentar a adesão ao tratamento medicamentoso, além da educação em saúde sobre as consequências decorrentes de uma pressão arterial descontrolada. Fica evidente que os idosos valorizam iniciativas educativas a partir de reuniões do programa HIPERDIA (embora não haja uma ênfase nas necessidades individuais, o acolhimento seja inadequado e a linguagem utilizada precise ser reformulada) já que recebem orientações que ampliam o horizonte e o conhecimento, possibilitando a mudança de hábitos outrora mantidos por falta de informação. Por outro lado, se faz necessário conhecer profundamente a população atendida pela equipe de Estratégia Saúde da Família (ESF), e a partir disso fazer a discussão de ações educativas emancipadoras e participativas.

Os estudos demonstram que a maior parte dos cadastrados no HIPERDIA são do sexo feminino e possuem baixa renda e escolaridade. Logo, deve haver um esforço das autoridades públicas acerca desse público em especial, seja através de campanhas, cursos, visitas domiciliares, dentre outras formas de informação, a fim de que se possa melhorar os índices ao qual se propõe o programa. Acrescenta-se a importância de novos e contínuos estudos para averiguar a realidade de outras localidades e a atuação específica da ESF e do HIPERDIA, e, a partir da identificação das reais necessidades locais, ir sanando as lacunas existentes.

Participação dos autores: Álef Lamark Alves Bezerra: Participou na contemplação de todos os elementos textuais para a construção do artigo, adaptação para o método Vancouver, adaptação para as normas da revista, revisão requerida pela revista, revisão textual e ortográfica. Daniel Sarmento Bezerra: Participou na contemplação de todos os elementos textuais para a construção do artigo, revisão textual, resumo e abstract. Danielle Serafim Pinto: Orientação para a construção do artigo e revisão textual. André Ricardo Bezerra Bonzi: Participou na discussão dos resultados do artigo, revisão textual. Ricardo Montenegro Nóbrega de Pontes: Revisão textual, revisão requerida pela revista. José Artur de Paiva Veloso: Orientação para a construção do artigo, revisão textual e ortográfica.

\section{REFERENCIAS}

1. Chaves AS, Santos AM, Alves, MTSSB, Salgado Filho, N. Associação entre declínio cognitivo e qualidade de vida de idosos hipertensos. Rev Bras Geriatr Gerontol. 2015;18(3):545-56. http://dx.doi.org/10.1590/18099823.2015.14043.

2. França DJR, Nunes JT, Fernandes MNF. As contribuições do cuidado ao idoso no programa de HIPERDIA, para a formação profissional. Rev Kairós Gerontol. 2014;17(2):31527. Disponível em: https://revistas.pucsp.br/index.php/kairos/ article/view/21750/16024.

3. Reticena KO, Piolli KC, Carreira L, Marcon SS, Sales CA. Percepção de idosos a cerca das atividades desenvolvidas no Hiperdia. Rev Min Enferm. 2015;19(2):107-13.

4. Silveira J, Scherer F, Deitos A, Bosco SMD. Fatores associados à hipertensão arterial sistêmica e ao estado nutricional de hipertensos inscritos no programa Hiperdia. Cad Saude Coletiva. 2013;21(2):129-34. http://dx.doi. org/10.1590/S1414-462X2013000200005.

5. Souza CS, Stein AT, Bastos GA, Pellanda LC. Blood Pressure Control in Hypertensive Patients in the "Hiperdia Program": A Territory-Based Study. Arq Bras Cardiol. 2014;102(6):571-8. doi: 10.5935/abc.20140081.

6. Focchesatto A, Rockett FC, Perry IDS. Fatores de risco e proteção para o desenvolvimento de doenças crônicas em população idosa rural do Rio Grande do Sul. Rev Bras Geriatr Gerontol. 2015;18(4):779-95. http://dx.doi.org/10.1590/18099823.2015.14150.
7. Gus I, Ribeiro RA, Kato S, Bastos J, Medina C, Zazlavsky. et al. Variações na Prevalência dos Fatores de Risco para Doença Arterial Coronariana no Rio Grande do Sul: uma análise comparativa entre 2002-2014. Arq Bras Cardiol. 2015;105(6):0-0. Disponível em: http://www.arquivosonline. com.br/2015/aop/AOP_6857.pdf.

8. Carvalho ALM, Leopoldino RWD, Silva JEG, Cunha CP. Adesão ao tratamento medicamentoso em usuários cadastrados no Programa Hiperdia no município de Teresina (PI). Cienc Saude Coletiva. 2012;17(7):1885-2. http://dx.doi. org/10.1590/S1413-81232012000700028.

9. Soar C. Prevalência de fatores de risco cardiovascular em idosos não institucionalizados. Rev Bras Geriatr Gerontol. 2015;18(2):385-95. http://dx.doi.org/10.1590/18099823.2015.14072.

10. Santos MIPO, Griep RH. Capacidade funcional de idosos atendidos em um programa do SUS em Belém (PA). Cienc Saude Coletiva. 2013;18(3):753-61. http://dx.doi.org/10.1590/ S1413-81232013000300021.

Recebido em: 20.06.17

Aceito em: 04.12.17 